\title{
Hinweise für Autorinnen und Autoren
}

Die wissenschaftliche Vierteljahreszeitschrift „Medien \& Kommunikationswissenschaft“ (bis Ende 1999 „Rundfunk und Fernsehen - Zeitschrift für Medien- und Kommunikationswissenschaft") wird seit 1953 vom Hans-Bredow-Institut herausgegeben und redaktionell betreut. Die Zeitschrift ist ein interdisziplinäres Forum für theoretische und empirische Beiträge aus der gesamten Medien- und Kommunikationswissenschaft. Für die Publikation in „Medien \& Kommunikationswissenschaft" kommen wissenschaftliche Beiträge in Betracht, die:

- ein Moment originärer theoretischer Leistung beinhalten bzw. einen theoretisch weiterführenden Argumentationsgang bieten;

- Befunde zu einem ausgewiesenen Problem von theoretischer oder medienpraktischer Relevanz darstellen;

- innerhalb eines wissenschaftlichen Diskurses Position beziehen und die Diskussion voranbringen können oder

- Literatur bzw. ausgewählte Literatur zu bestimmten Problemstellungen systematisch und vergleichend zusammenfassen und eine Übersicht über den Stand der Theorie und/oder Empirie geben.

Die Redaktion bietet außerdem die Möglichkeit zur Stellungnahme und Erwiderung zu publizierten Beiträgen. Stellungnahmen und Erwiderungen, die den in „Medien \& Kommunikationswissenschaft“ üblichen inhaltlichen und formalen Standards entsprechen und geeignet sind, die wissenschaftliche Diskussion zu fördern, werden im nächstmöglichen Heft publiziert. Die Redaktion räumt dabei dem Autor bzw. der Autorin des Beitrages, auf den sich die Stellungnahme bezieht, die Möglichkeit einer Erwiderung ein.

Manuskripte, die zur Publikation in „Medien \& Kommunikationswissenschaft“ eingereicht werden, dürfen nicht anderweitig veröffentlicht sein und bis Abschluss des Begutachtungsverfahrens nicht anderen Stellen zur Veröffentlichung angeboten werden.

Im Sinne der Förderung des wissenschaftlichen Diskurses und der kumulativen Forschung sowie der Qualitätssicherung legt die Redaktion bei der Begutachtung von Beiträgen besonderen Wert darauf, dass größtmögliche Transparenz hinsichtlich der verwendeten Daten hergestellt wird. Autorinnen und Autoren empirischer Beiträge verpflichten sich mit der Einreichung des Manuskripts, dass sie die Art und Weise der Datenerhebung bzw. den Zugang zu Datenbeständen, die von Dritten (z. B. Datenbanken) zur Verfügung gestellt worden sind, ausreichend dokumentieren, um so die Voraussetzungen für Sekundäranalysen und Replikationen zu schaffen. Zugleich erklären sie sich bereit, die verwendeten Daten bei wissenschaftlich begründeten Anfragen im Rahmen der jeweils gegebenen Möglichkeiten für weitere Analysen zur Verfügung zu stellen.

Im Hinblick auf eine transparente Dokumentation des methodischen Vorgehens entwickeln sich zunehmend standardisierte Kriterienkataloge. Dies ist in der Kommunikationswissenschaft insbesondere für die standardisierte Inhaltsanalyse der Fall. Aufsätze, in denen standardisierte Inhaltsanalysen dargestellt werden, sollten folgende Angaben enthalten: Grundgesamtheitsdefinition, Stichprobendesign, Aufgreifkriterium (inkl. Angabe der Identifikationsreliabilität), Design des Reliabilitätstests (Art der Reliabilitätsberechnung, Art der Stichprobe und Stichprobenumfang, Zeitpunkt, Zahl der Codierer, Begründung der Koeffizientenwahl), Zahl der bei der Codierung eingesetzten Codierer, Zahl der Ausprägungen und vorzugsweise zufallskorrigierte Reliabilitätskoeffizienten für alle dargestellten Variablen, Bewertung und Konsequenzen des Reliabilitätstests. Aufsätze, in denen andere Methoden dargestellt werden, sollten sich in diesem Sinne an den der jeweiligen Methode entsprechenden Kriterien orientieren. 


\section{Formalien:}

- Manuskripte sind der Redaktion im Word-Format per E-Mail zuzuschicken.

- Da die eingereichten Manuskripte anonymisiert begutachtet werden, sind zwei Titelblätter erforderlich: eines mit Angabe des Titels und der Namen und Anschriften der Autorinnen und Autoren, eines ohne Anführung der Namen und Adressen. Das Manuskript selbst darf keine Hinweise auf die Autorinnen und Autoren enthalten.

- Beizufügen ist eine kurze Zusammenfassung des Beitrags (max. 15 Zeilen), die dem Leser als selbständiger Text einen hinreichenden Eindruck vom Inhalt des jeweiligen Beitrags vermittelt.

- Der Umfang der Beiträge darf 20 Manuskriptseiten (55.000 Buchstaben inkl. Leerzeichen) nicht überschreiten.

- Gliederung des Textes: Jedes Kapitel und Unterkapitel sollte mit einer Überschrift (in Dezimalzählung) versehen sein.

- Hervorbebungen im Text sind kursiv oder fett zu kennzeichnen.

- Hinweise und Literaturbelege sollen erfolgen durch Angabe von Autor, Erscheinungsjahr und Seitenziffer im fortlaufenden Text - z. B.: . . . (Müller 1990: 37) . . -, wobei der vollständige bibliographische Nachweis über ein Literaturverzeichnis im Anschluss an den Beitrag ermöglicht wird.

- Aufgrund der in verschiedenen Fachtraditionen unterschiedlichen Zitierregeln gibt M\&K für das Literaturverzeichnis keine bestimmte Variante vor. Die einzige Vorgabe besteht darin, dass alle erforderlichen Literaturangaben vorhanden sind und dass diese für das gesamte Manuskript konsistent gestaltet werden.

Über eine Annahme des Manuskripts und den Zeitpunkt der Veröffentlichung entscheidet die Redaktion auf der Grundlage mehrerer Gutachten. Dem/der Autor/in wird die Redaktionsentscheidung schriftlich mitgeteilt. Im Falle einer Entscheidung für Überarbeitung, Neueinreichung oder Ablehnung legt die Redaktion die Gründe für ihre Entscheidung offen. Dazu werden die anonymisierten Gutachten, evtl. auch nur in Auszügen, zugesandt. Das Begutachtungsverfahren ist in der Regel sechs Wochen nach Eingang des Manuskripts abgeschlossen; falls die Begutachtung längere Zeit erfordert, werden die Autor/inn/en benachrichtigt.

Von jedem Originalbeitrag werden 20 Sonderdrucke kostenlos zur Verfügung gestellt. Weitere Sonderdrucke können bei Rückgabe der Fahnenkorrektur an die Redaktion schriftlich gegen Rechnung bestellt werden.

Verlag und Redaktion haften nicht für Manuskripte, die unverlangt eingereicht werden. Mit der Annahme eines Manuskripts erwirbt der Verlag von den Autorinnen und Autoren alle Rechte, insbesondere auch das Recht der weiteren Vervielfältigung zu gewerblichen Zwecken im Wege des fotomechanischen oder eines anderen Verfahrens.

\section{Herausgeber:}

Hans-Bredow-Institut, vertreten durch das Direktorium Prof. Dr. Uwe Hasebrink und Prof. Dr. Wolfgang Schulz

\section{Schriftleitung:}

Christiane Matzen, M.A. (ViSdP), Hans-Bredow-Institut | Heimhuder Str. 21 | 20148 Hamburg

Telefon 040/450217-41 | E-Mail c.matzen@hans-bredow-institut.de

Druck und Verlag:

Nomos Verlagsgesellschaft mbH \& Co. KG | Waldseestraße 3-5 | 76530 Baden-Baden,

Telefon 07221/2104-0| Fax 07221/2104-27

Anzeigen:

sales friendly Verlagsdienstleistungen | Siegburger Str. 123 | 53229 Bonn

Telefon 0228/978980 | Fax 0228/9789820 | E-Mail roos@sales-friendly.de 